\title{
The use of partial temporal information in dating personal events
}

\author{
CHARLES P. THOMPSON \\ Kansas State University, Manhattan, Kansas \\ JOHN J. SKOWRONSKI \\ Ohio State University, Newark, Ohio \\ and \\ ANDREW L. BETZ \\ Ohio State University, Columbus, Ohio
}

\begin{abstract}
The use of different types of partial temporal information is shown to affect dating accuracy and the distribution of errors in event dating. Several different types of partial temporal information are discussed, but three are highlighted by the data. Specifically, subjects' dating error patterns suggest that they (1) use different types of within-week information, (2) use recalled event sequences, and (3) use boundary landmarks to report the dates of events. In general, these data suggest that although precise temporal information is sometimes represented in the memory trace for an event, more often the date-related information is inferred from other aspects of memory.
\end{abstract}

Evidence from many sources suggests that when asked to remember the exact date of an event from their personal history, people often cannot do so (Brewer, 1988; Huttenlocher, Hedges, \& Bradburn, 1990; Thompson, Skowronski, \& Lee, 1988a; Wagenaar, 1986). However, people do not often randomly guess at dates either (Thompson et al., 1988a). Instead, they frequently use partial temporal information to narrow the possible dates of occurrence. For example, when asked to provide a date for an event, an individual might remember only that the event occurred last summer. Despite the fact that her attempt at dating the event will likely be erroneous, the partial date-relevant knowledge that she does possess (it happened in the summer) should enable her to reduce the range of error in the provided estimate.

Several different kinds of partial temporal information may be accessed in the process of reconstructing an event's date. For example, partial temporal information can potentially be derived from (1) the accessibility of the event in memory (Bradburn, Rips, \& Shevell, 1987; Brown, Rips \& Shevell, 1985); (2) the details of the event itself (Skowronski, Betz, Thompson, \& Shannon, 1991); (3) world knowledge or prototypic knowledge that allows a reasonable inference about some aspect of time (Thompson et al., 1988a); (4) recalled temporal relationships between events (Loftus \& Marburger, 1983), including events that form boundaries for dating periods (Hutten-

We thank the assistants who helped collect and tabulate the data: Margaret Van Balen, Jean Isham, Laura Shannon, and W. Richard Walker. Drew Betz is now at Boise State University. Reprint requests should be sent to C. P. Thompson, Department of Psychology, Bluemont Hall, Kansas State University, Manhattan, KS 66502. locher, Hedges, \& Prohaska, 1988); and (5) temporal details or "tags" that are part of one's recalled knowledge about an event (Skowronski, Betz, Thompson, Walker, \& Shannon, in press).

Although the evidence indicates that people use these different types of partial temporal information in their dating efforts, we do not have very specific knowledge about the frequencies with which these types of information are employed, or about the specific effects that the different types of partial knowledge have on the distribution of dating errors. In the present study, we investigated these issues by asking subjects to date events from their own personal histories, and by looking at factors affecting dating accuracy and the pattern of errors in the dates provided. More specifically, the data reported in the present paper are used to address four issues that are relevant to the use of partial temporal information to date events.

First, we look at the relationship between subjects' selfreported dating strategies and dating accuracy. On the basis of the results provided by Thompson et al. (1988a), we believe that the strategies that subjects use to date events should be at least partially, and perhaps entirely, determined by the temporal information that can be recalled or reconstructed. Furthermore, this recalled or reconstructed information should affect the accuracy of dating attempts made with those strategies. The data in the present paper will allow us to look at the relation between dating strategies and accuracy, and to gather evidence on the frequency of usage of various dating strategies.

Second, we have previously argued that people sometimes recall the day of the week on which an event occurred in order to help them date that event (Skowronski et al., 1991; Skowronski et al., in press). In the present 
paper, we expand this idea by suggesting that prototypic or event-derived knowledge about the time of week (weekday vs. weekend, midweek, etc.) in which an event $o c-$ curred will also affect event dating. In pursuing this hypothesis, we develop a theoretical model that uses four sources of within-week information to predict a specific pattern of dating errors, and we look for this pattern in our date estimation data.

Third, we investigate the hypothesis that subjects use recalled event sequences as an aid to date estimation. That is, we believe that people can recall an event sequence, or can reconstruct from world knowledge what the likely event sequence would have been. Once a sequence is established, constructing or recalling a date for only one of the events helps establish dates for all of the events in the sequence. If the sequenced events occur on consecutive days, then when the data are examined in the order of their occurrence, one consequence should be the existence of runs of events with the same dating error. We examine the data to see whether these runs occur in the dating protocols at a greater than chance level.

Finally, we will examine evidence related to the proposal made by other investigators (Huttenlocher et al., 1990; Huttenlocher et al., 1988; Rubin \& Baddeley, 1989) that the knowledge that there are upper and lower bounds to an event's age can produce telescoping and time expansion effects in event dating. These authors have proposed that both telescoping (dating an event as more recent than its actual occurrence) and time expansion (dating an event as earlier than its actual occurrence) can be partially explained by the fact that error direction is naturally limited near a boundary. In the present study, we varied the boundary for two different groups of subjects and evaluated the effects of this factor on telescoping and time expansion tendencies.

\section{METHOD}

\section{Subjects}

The subjects in this study were 33 undergraduates at Kansas State University (KSU) and 30 undergraduates at Ohio State University, Newark (OSUN). All received class credit for their participation.

\section{Materials}

The materials, which were recorded by the subjects, consisted of unique personal events that occurred during the academic term. The subjects recorded one event each day, and the event records were collected weekly. The subjects were instructed that there were three restrictions on the events: (1) the events had to be unique (they were expected to occur no more than once during that semester); (2) the events could not be overly embarrassing; and (3) the events were to be described in three written lines or fewer.

\section{Procedure}

The 33 subjects from KSU generated events for approximately 14 weeks, and the 30 subjects from OSUN generated events for approximately 10 weeks.

The subjects were individually tested over the content of their diaries sometime during the 14 th or 15 th week (KSU) or during the 9th or 10th week (OSUN) of the academic term. During the test, the events were read to each subject in a random order of presentation. In responding to each event, the subject first determined whether or not the event was unique. This procedure was used because the subjects reasonably might not be able to provide a specific date for events that occurred more than once during the academic term. The subjects had no problem making the uniqueness judgment. If an event was not unique, it was deleted, and the subject went on to the next event. If the event was judged to be unique, the subject then rated the event on several dimensions irrelevant to the present paper

After giving the ratings, the subjects estimated the date of occurrence of the event. As an aid to date estimation, the subjects were given standard calendar pages for the general period in which the diaries were kept. These calendar pages contained no extraneous date-related information (i.e., no holidays, no indication of the beginning of the term, etc.), only the day of the week and the date. After estimating the date, the subjects reported the subjective accuracy of their estimate by stating a range of error (e.g.. "within 2 days," "within 6 days") for that estimate.

Finally, the subjects specified the strategy they used to determine the date of the event. The subjects were given a list of seven possible strategies plus a category designated as other. The seven strategies listed were derived from a previous study (Thompson et al., 1988a). The strategies were as follows: (1) exact date was known; (2) specific reference to another event; (3) general reference-the general time period (e.g., summer) was known; (4) estimated number of intervening events since the event being dated; (S) clarity of memory; (6) prototypic date-knew day, week, or month (e.g., always bowl on Wednesdays); and (7) guess.

\section{RESULTS AND DISCUSSION}

\section{Subject Strategy, Partial Temporal Information, and Dating Accuracy}

We argue that subjects' dating strategies and the accuracy of dates produced by using those strategies should be related, in part because of the different types of partial temporal information that are available for use in each strategy. Because of differences in the temporal precision implicit in different types of memories, some types of strategies should naturally produce more accurate estimates than should other strategies. For example, subjects should generally exhibit much more accuracy in dating if they can remember the exact week in which an event occurred than if they can only remember the season in which the event occurred, or if they only can remember that the event happened sometime after a landmark (such as the Desert Storm invasion).

To investigate this idea, we first calculated the absolute values of the dating errors for each event reported by each subject. We then entered the linear effect of age, the quadratic effect of age, subject, and dating strategy as predictors in a pooled within-subject regression analysis of these dating errors.

The results of the analysis indicated that dating strategy was a significant predictor of dating accuracy $\left[F(6,4478)=73.71, M S_{\mathrm{e}}=89.94, p<.001\right]$. The mean absolute dating errors, presented in Table 1, indicate that there was a systematic relation among the strategies in terms of the accuracy of estimates produced by those strategies. Unsurprisingly, subjects' estimates were most accurate when they thought they remembered the exact date, but dating accuracy was also fairly good when subjects dated an event in reference to other events.

As one might expect, strategies that involved the use of other types of partial temporal information produced 
Table 1

Relationships Between Dating Strategy Used and Absolute Value of Dating Errors (in Days)

\begin{tabular}{lcc}
\hline \multicolumn{1}{c}{$\begin{array}{c}\text { Dating } \\
\text { Strategy }\end{array}$} & $\begin{array}{c}\text { Frequency of } \\
\text { Reported Use }\end{array}$ & $\begin{array}{c}\text { Mean Error } \\
\text { (Absolute Value) }\end{array}$ \\
$\begin{array}{l}\text { Exact date recalled } \\
\text { Related to } \\
\text { reference event }\end{array}$ & 802 & 1.30 \\
$\begin{array}{c}\text { General reference } \\
\text { period recalled }\end{array}$ & 1,011 & 4.08 \\
$\begin{array}{l}\text { Estimated number of } \\
\text { intervening events }\end{array}$ & 1,312 & 8.93 \\
$\begin{array}{l}\text { Clarity of recall } \\
\text { Generated prototypic } \\
\text { temporal information }\end{array}$ & 182 & 10.74 \\
Guess & 210 & 11.15 \\
\hline
\end{tabular}

less accurate estimates. Remembering the general reference period, estimating the number of intervening events, using memory clarity to estimate dates, and prototypic dating (remembering the day, week, or month) produced dates of intermediate accuracy. Guessing yielded the lowest level of dating accuracy.

It should be noted that in the present study, we asked the subjects to report only one dating strategy, which presumably was the dominant strategy that they employed. However, it is not necessarily true that subjects employ only one strategy to date events. When given the opportunity, subjects will often report that they have used multiple strategies to date a single event (Thompson et al., 1988a). Because the frequencies in Table 1 likely reflect subjects' use of each strategy when it was dominant, these frequencies probably underestimate the overall rate of use of the strategies, particularly the strategies that do not involve recall of the exact date.

\section{Time of Week}

As noted above, in one strategy for dating events, subjects can employ prototypic information: the typical day, week, or month in which an event occurs. For example, if an event involves the bridge club, subjects can guess that the event occurred on Saturday because that is their bridge night. Thus, subjects might not know the date on which an event occurred, but might be able to infer the day on which it occurred.

The day of the week on which an event occurred can also be directly recalled. That is, subjects might have temporal information encoded along with the memory of the event. Hence, subjects might directly remember that an event occurred on a Monday.

Because we collect the diaries over a relatively short time interval of approximately 3-4 months, the day of the week on which an event has occurred is a particularly appropriate object of study in our diary memory experiments. In that short interval, we think that much of the available partial information about dates of events (but, as indicated by the frequency data, certainly not all available partial temporal information) will be about the day of the week on which the event occurred. This hypothesis has received support from our previous research (Skowronski et al., 1991; Skowronski et al., in press).
In the present paper, we examine the possibility that there are, in fact, four different types of within-week information that an individual may have available for dating events. We do not exclude other types of information about the week, but we believe that these four types are applicable, in principle, to all individuals.

In the discussion that follows, it is convenient for us to use modulo arithmetic to express dating error. A number in modulo arithmetic is expressed as a remainder. Thus, $0 \bmod 7$ refers to $0,7,14$, etc. Similarly, $2 \bmod$ 7 refers to $2,9,16,23$, etc.

We begin our discussion with the simplest case, that in which the day of the week is known. For example, an event may involve bowling, and the individual knows that he bowls on Tuesday evenings. Thus, he will select a Tuesday as the day for that event. If he has correctly recalled or reconstructed the day of the week, either the date will be exact, or the error in dating will be a multiple of 7 days. Accordingly, for the events for which the subject knows the day of the week, all errors will be $0 \bmod 7$. That result is shown in Table 2 , with $100 \%$ of the cases at $\mathbf{0} \bmod 7$.

Now consider the set of events for which the subject knows that the event occurred on the weekend, but cannot exactly recall whether it occurred on Saturday or Sunday. Random selection of the day will result in $50 \%$ of the dating estimates falling on the correct day $(0 \bmod 7)$. The remaining estimates will be split evenly (i.e., $25 \%$ each) between a day long $(1 \bmod 7)$ and a day short $(6$ mod 7). That result is also shown in Table 2.

Similar arguments can be developed for knowing that the event occurred during the midweek (Tuesday, Wednesday, or Thursday) or sometime during the typical work week (a "week day"-Monday through Friday). The logical consequences of those arguments, expressed in percentages, are also shown in Table 2.

Because we do not know the relative frequency with which these bits of partial information are used, for purposes of this model we make the simplifying assumption that they occur equally often. Thus, the last line of Table 2 gives the mean of the expected relative percent distribution of dating errors caused by estimation processes, assuming that subjects use the four bits of partial information with equal frequency.

Earlier, we noted that we had previously provided evidence (Skowronski et al., 1991) showing that subjects use

Table 2

Theoretical Percent Error Distribution Based on Using Four Types of Within-Week Partial Temporal Information

\begin{tabular}{lrrrrrrrr}
\hline \multicolumn{1}{c}{\begin{tabular}{c} 
Partial \\
Information \\
\multicolumn{1}{c}{ Type }
\end{tabular}} & 0 & 1 & 2 & 3 & 4 & 5 & 6 \\
\hline & \multicolumn{1}{c}{ Error (Mod 7) } \\
Day of Week & 100 & 0 & 0 & 0 & 0 & 0 & 0 \\
Weekend & 50 & 25 & 0 & 0 & 0 & 0 & 25 \\
Midweek & 33 & 22 & 11 & 0 & 0 & 11 & 22 \\
Week Day & 20 & 16 & 12 & 12 & 12 & 12 & 16 \\
$M$ & 50.8 & 15.8 & 5.8 & 3 & 3 & 5.8 & 15.8 \\
\hline
\end{tabular}

Note-Percentages within a particular information type may not sum to 100 , because of rounding. 
the day of the week as partial information to aid in dating events. The inclusion of the three other within-week sources of partial temporal information is important because they can help to account for data that cannot be explained solely in terms of knowing the day of the week. Knowing the day of the week affects only errors at 0 mod 7. By contrast, adding the other three bits of partial information produces a curvilinear function affecting all points on the mod 7 distribution.

We assume that the mean distribution of errors developed in Table 2 is applied as a weighting factor to an underlying distribution of errors. We chose the underlying distribution on the basis of our knowledge of performance in dating events. Subjects are quite accurate in dating recent events, but they show increasing error as the retention interval increases (e.g., Skowronski et al., 1991; Thompson, 1982). However, subjects continue to date a portion of their events accurately throughout the retention interval. In short, absolute errors tend to accumulate at the zero error point and fall off fairly rapidly thereafter.

We believe that this performance is best modeled as a negative exponential curve. Specifically, we have modeled the underlying distribution of absolute errors to have the form

$$
a e^{-b(x+1)} \text {, }
$$

with $x=$ dating error, $a=28$ and $b=.11 .^{1}$ We do not believe that the parameters $a$ and $b$ are fixed. Instead, we would expect the parameters $a$ and $b$ to be affected by such characteristics as the length of the retention interval and the memorability of the events that are tested.

Deriving the final theoretical percent distribution involves several simple steps. First, each point in the underlying distribution is multiplied by the appropriate weight described in Table 2 . Then, we rescale the distribution to produce a percent distribution.

However, we do not rescale the entire distribution. Previous research (Thompson et al., 1988a) has indicated that, within this paradigm, subjects report knowing the exact date for about $10 \%$ of all events. ${ }^{2}$ Furthermore, subjects rarely make an error in dating an event when they report knowing the exact date. This implies that about $10 \%$ of the events are not subject to estimation biases. Therefore, we rescale the distribution to account for $90 \%$ of the distribution rather than $100 \%$ of the distribution. We complete the theoretical percent distribution by adding $10 \%$ (representing the proportion of dates assumed to be perfectly recalled) to the 0 error point. These procedures yield the theoretical percent distribution shown in Figure 1.

To provide data against which this theoretical distribution can be evaluated, we generated the distribution of absolute errors by pooling errors over all subjects and all events. Figure 1 depicts these data. As illustrated in this figure, the empirical distribution closely resembles the theoretical distribution. Note particularly the curvilinear function between 0 and 7 days error, as well as between 7 and 14 days error. As noted above, using only day of

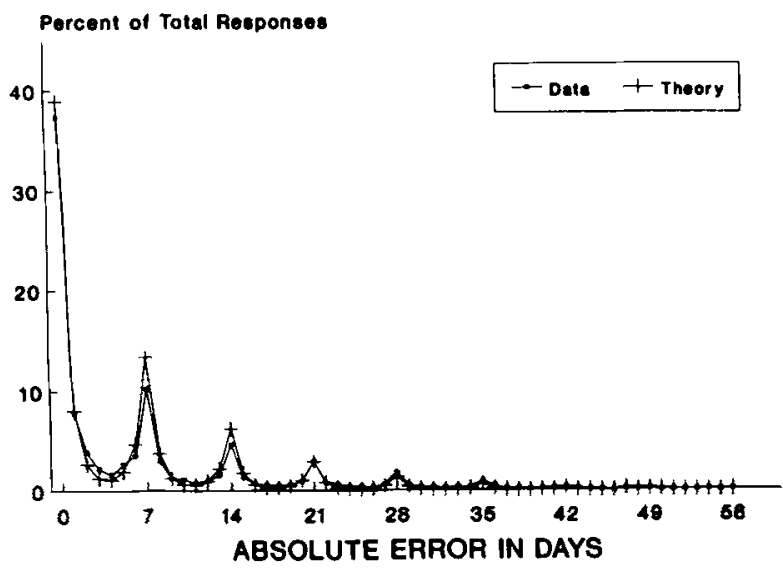

Figure 1. Empirical and theoretical distributions of absolute dating errors (in percent).

the week as partial information would affect errors only at $0 \bmod 7$ and would not produce the curvilinear functions shown between 0 and 14 days error in the empirical distribution.

To verify that the model generally applies to data from diary memory studies, we generated the absolute error distribution for two additional relatively recent data sets. The first data set was taken from the second experiment in Thompson (1985). From that data set, we generated the absolute error distribution for 24 subjects responding to events spanning a 12 -week retention interval. The second data set was taken from the first experiment in Thompson, Skowronski, and Lee (1988b). From that data set, we generated the absolute error distribution for 35 subjects, with events again spanning a 12-week retention interval. The results for both studies are presented in Table 3 . The theoretical predictions and the data from the present paper are also included for comparison.

It is clear that the theoretical model provides a good approximation to the results generated from the other two data sets. Furthermore, because this theoretical model was developed from assumptions about subjects' use of timeof-week information, the good collective fit of the three data sets provide support for the hypothesis that subjects use the four types of within-week information in reconstructing the dates of personal events.

In fact, the data suggest that some of this within-week information might be used more often than others. Close inspection of the means in Table 3 indicates that the model slightly overpredicts the error percentages at $0 \bmod 7$. The most reasonable explanation for this overprediction is that our simplifying assumption that subjects use the four types of within-week information equally often is incorrect. Instead, these data suggest that subjects might use day-of-week information a bit less often than the other three types. This idea could easily be investigated in future studies.

In fact, a direct assessment of the type of within-week information used for each date estimate is necessary for an important theoretical reason. It is possible that the data 
Table 3

Theoretical and Empirical Distributions (in Percent) of Errors in Dating Personal Events

\begin{tabular}{|c|c|c|c|c|}
\hline \multirow{2}{*}{$\begin{array}{l}\text { Absolute Error } \\
\text { (in Days) }\end{array}$} & \multirow{2}{*}{$\begin{array}{l}\text { Theory } \\
\text { Predicts }\end{array}$} & \multicolumn{3}{|c|}{ Experiment } \\
\hline & & 1 & 2 & 3 \\
\hline 0 & 38.93 & 37.30 & 29.58 & 34.42 \\
\hline 1 & 8.04 & 7.80 & 9.11 & 8.69 \\
\hline 2 & 2.63 & 3.90 & 3.93 & 3.27 \\
\hline 3 & 1.23 & 2.20 & 2.10 & 1.31 \\
\hline 4 & 1.10 & 1.60 & 2.02 & 1.37 \\
\hline 5 & 1.89 & 2.60 & 3.04 & 1.89 \\
\hline 6 & 4.64 & 3.50 & 3.52 & 2.61 \\
\hline 7 & 13.39 & 10.30 & 11.82 & 12.21 \\
\hline 8 & 3.72 & 3.00 & 3.16 & 3.20 \\
\hline 9 & 1.22 & 1.20 & 1.62 & 1.11 \\
\hline 10 & .57 & 1.10 & .85 & .91 \\
\hline 11 & .51 & .70 & .97 & 1.05 \\
\hline 12 & .88 & 1.00 & 1.34 & .72 \\
\hline 13 & 2.15 & 1.50 & 1.50 & 1.63 \\
\hline 14 & 6.20 & 4.60 & 5.63 & 5.42 \\
\hline 15 & 1.72 & 1.30 & 1.66 & 1.83 \\
\hline 16 & .56 & .70 & 1.34 & 1.18 \\
\hline 17 & .26 & .50 & .57 & .39 \\
\hline 18 & .24 & .50 & .61 & .26 \\
\hline 19 & .41 & .60 & .85 & .65 \\
\hline 20 & .99 & .90 & 1.09 & 1.18 \\
\hline 21 & 2.87 & 2.90 & 3.04 & 2.55 \\
\hline 22 & .80 & .70 & .93 & .59 \\
\hline 23 & .26 & .60 & .40 & .72 \\
\hline 24 & .12 & .40 & 40 & .46 \\
\hline 25 & .11 & .40 & 16 & .20 \\
\hline
\end{tabular}

Note-Experiment 1 is the data from the experiment in this paper. Experiment 2 is the data from the first experiment in Thompson et al. (1988b). Experiment 3 is the data from the second experiment in Thompson (1985).

observed in Figure 1 and Table 3 are not due to the subjects' use of the four types of within-week information, but instead reflect random error in the subjects' use of day-of-the-week information. ${ }^{3}$ To put this in concrete terms, these data could simply reflect the fact that subjects are somewhat likely to misidentify a Sunday event as occurring on either a Saturday or a Monday, are less likely to misidentify that event as occurring on a Friday or Tuesday, and are even less likely to misidentify that Sunday event as occurring on a Thursday or Wednesday. If these misidentification error patterns occurred frequently enough, one would expect data very similar to those depicted in Figure 1 and Table 3.

This is a highly plausible argument, and it cannot be discounted without more precise information about the within-week cues used by our subjects. That is, to discriminate between these two explanations, it is necessary to assess the exact within-week temporal information subjects use to construct a date estimate, how often they use that type of within-week temporal information, and the nature of the error pattern associated with that within-week information. The present study did not assess these specifics.

However, our observations of subjects' on-line verbal protocols during the dating task give us reason to believe that subjects do make use of various kinds of within-week cues. Our subjects frequently appeared to know with a high degree of certainty whether an event (i.e., a party)

was on a weekend or was sometime during the workweek (i.e., a class or an exam), even when they did not know the exact day of the week. Although these observations do not conclusively rule out an explanation based on random error, they do strongly suggest that subjects were accessing these types of within-week information when constructing their date estimates.

Another theoretical issue that should be mentioned concerns the use of "rounding" in date estimation. Huttenlocher et al. (1990) provided data indicating that subjects' date estimates are often biased by the use of prototypic values (e.g., subjects tend to use rounded output values: $2,3,5,10,15$, etc.). The data from all three studies presented in Table 3 do not show any evidence of such rounding effects.

It should be obvious that the differences between our results and the results of Huttenlocher et al. (1990) are caused by methodology. We essentially ask subjects to pinpoint a calendar date for an event; Huttenlocher et al. ask subjects to report how long ago a date occurred. The fact that we ask for exact dates makes it difficult for subjects to use the prototypic output values that are evident in Huttenlocher et al.'s data. One important conclusion from the present data is that how you ask the dating question (what date vs. how long ago) will affect the pattern of dating errors obtained.

The methodological explanation for the different results generated by the two paradigms is bolstered by the fact that one aspect of our data does show "rounding" effects: subjects' accuracy estimates. As is illustrated in Figure 2, the subjects did tend to use prototypic values when reporting their confidence estimates. Although the "weekly" peaks at $0,7,14$, and 21 days are similar to those observed in the error data depicted in Figure 1, there are most definitely estimation peaks (notably at 5,10 , and 30 days) that do not appear in the date error data, and these likely represent the subjects' use of rounded values in reporting their confidence judgments.

The difference in assessment methodology used in the two paradigms might have other implications as well. As

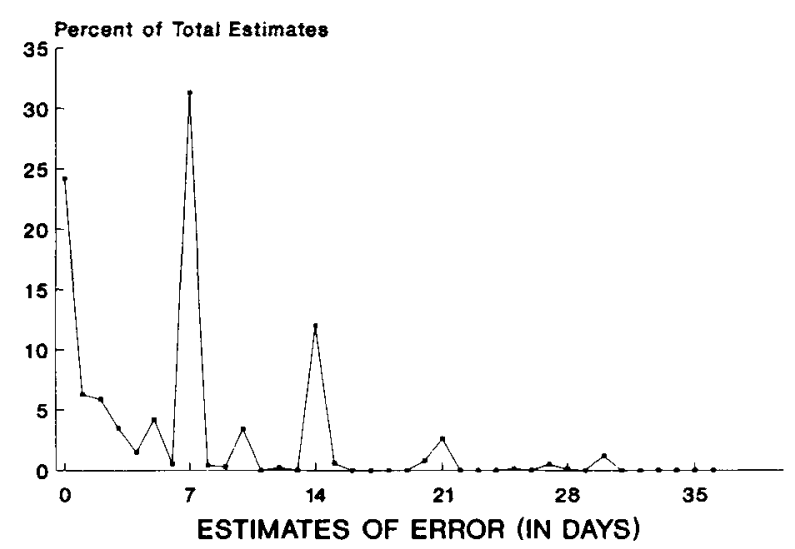

Figure 2. Distribution of subjects' estimates of error ranges for event dates (in percent). 
we have suggested elsewhere (Skowronski et al., in press), the two different dating questions commonly used to assess dating (how long ago vs. what date) might cause subjects to engage in memory searches of differing depths. In responding to the how long ago question, subjects might search memory just enough to provide a reasonably accurate dating value-a value that can be expressed in terms of a prototypic response. By comparison, the what date question intuitively demands more precision and might induce subjects to engage in a more detailed search of memory.

This reasoning suggests that a more intensive comparison of the accuracy and error patterns produced by the two types of dating questions within the same study might provide insight into the role that memory processes play in date estimation. For example, we wonder if the data so compellingly depicted in Figure 1 and Table 3 would emerge with the how long ago question. More specifically, if our results are dependent on subjects' use of partial within-week temporal knowledge, and if this partial temporal knowledge is not used in responding to the how long ago question, one might not observe the "peaked" pattern in subjects' estimates of how long ago events occurred.

Finally, it should be noted that direct comparisons between the data we obtained and those produced by Huttenlocher et al. (1990) are complicated by the fact that, in our paradigm, subjects are provided with more memory cues (i.e., the blank calendar) than are provided in the Huttenlocher et al. paradigm. It is possible that the "peaked" error pattern might emerge with the how long ago question if a calendar were provided as a dating aid. More research is clearly needed to disentangle the effects of question type and dating aids on event dating errors.

\section{Events in a Sequence}

Many events in our lives are part of a sequence of events with a central theme. There are themes with relatively long time frames, such as being a parent or attending college. There are also themes with fairly short time frames, such as preparing for a large party or shopping for a new apartment.

In coding diaries for previous studies, it had been noted that sequenced entries of thematically related events would occur with some frequency. For example, consider a sequence of diary entries taken from one of the diaries collected and used in Skowronski et al.'s (1991) study (identifying names are deleted):

Sunday: Husband got the keys to our new apartment today. Monday: Cleaned the new apartment all day.

Tuesday: Spent the first night in our apartment-we were really tired.

Wednesday: Aunt $Y$ came to our new apartment today for the first time. She brought us a strawberry cheesecake.

A series of related events such as the series listed above might contain one or more events that could reasonably be used as an anchor to date other events. Following the reasoning of Loftus and Marburger (1983), we think that one useful strategy in dating events from a series with a central theme would be to assign a date to the most datable (landmark) event and then use it as a reference point in dating other events.

Loftus and Marburger (1983) argued that dating with reference to a landmark event could lead to increased dating accuracy, and the data they collected indicated that this sometimes occurs. However, the use of this strategy also has implications for other characteristics of the error distribution. If subjects use an anchor event to date other events in the sequence, their dating protocols should show evidence of runs of events that are in error by a systematic amount (i.e., the error of the reference event). Not coincidentally, the four events that were described above were all dated incorrectly and were all thought to have occurred 22 days more recently than they had actually occurred.

Researchers investigating memory have already constructed several measures to determine whether such runs exist in a data set at a level above that expected by chance. For example, there is a measure called the adjusted ratio of clustering (ARC), which was developed to measure organization in memory (Roenker, Thompson, \& Brown, 1971) by looking at output clustering. An ARC score is derived from the number of times that items within the same category occur in sequence (a run), and the number of runs expected by chance. An ARC score of zero (0) represents a chance level of runs in a data set; an ARC score of one (1) represents the maximum level of runs in a data set.

After arranging the data so that the events were in the order in which they originally appeared in the diaries, we applied the ARC measure to the raw (signed) dating errors produced by our subjects. For this calculation, we treated each different error value as a separate category. Because subjects often had a high probability of reporting the exact date of an event for the most recent events, a circumstance that could artificially inflate the ARC score, in our calculation of each subject's ARC score we ignored each subject's data for the most recent 2-week period. The mean ARC score calculated from these data was reliably above zero $[M=.097$, one-tailed $t(63)=$ 6.97, $\left.S E_{\mathrm{M}}=.014, p<.0001\right]$.

By the standards of classical memory research, this ARC score is low. To provide a comparison, ARC scores in the recall of categorized lists are about .80 after a few recall trials (see, e.g., Thompson \& Roenker, 1971). However, given that using related events in a series for purposes of dating events is just one of an array of strategies that are brought to bear, and given that thematic runs of related events do not dominate the diary entries, a relatively modest ARC score might be all that can be expected.

These conjectures are supported by the strategy frequency data reported in Table 1 . As the data in that table indicate, subjects reported using a relational strategy to date events only about $22 \%$ of the time. Given that relational dating is even possible for a minority of the events, it is not surprising that the overall clustering index is relatively modest. Nonetheless, the fact that the clustering in- 
dex is significantly above chance levels supports the idea that a relational strategy is sometimes used in event dating, and sometimes causes subjects to make runs of systematic, equivalent dating errors.

\section{The Effects of Boundaries on Telescoping and Time Expansion}

The dating attempts of subjects in our diary studies might be influenced by the partial temporal information provided by reference events in another important way. Both the upper bound of the retention interval (i.e., the date on which they began the diary study) and the lower bound on the retention interval (i.e., the diary test date) provide landmark events that might impact event dating. More specifically, as suggested in several recent papers (Huttenlocher et al., 1990; Huttenlocher et al., 1988; Rubin \& Baddeley, 1989), the effect of boundary knowledge (or bounding) may be to produce apparent telescoping (age underestimation) and time expansion (age overestimation).

The manner in which boundaries can produce overall evidence of telescoping in a data set can be illustrated by considering a case in which an individual, at the end of summer, makes an estimate of the date of an event known to have occurred during the summer. An event occurring in early summer can have the full range of telescoping errors, but the range of time expansion errors is limited by the beginning-of-summer boundary. Thus, events near the oldest possible date boundary will tend to show telescoping effects. The reverse situation should occur at a recent boundary. An event occurring yesterday can have the full range of time expansion errors, but can only have limited telescoping. Thus, events at a recent boundary should show evidence of time expansion. Because of the nature of memory processes, date estimates for recent events should be more accurate than date estimates for old events, so that boundary-related telescoping will be greater than boundary-related time expansion. Averaging across all errors will yield apparent overall evidence of telescoping.

One implication of the boundary hypothesis is that the amount of telescoping at any retention interval will depend on the location of the oldest-date boundary. That is, because the error distribution is increasingly truncated as one approaches the oldest-date boundary, the closer an event is to the boundary, the more that event's date should be telescoped. One implication of this is that telescoping is not dependent on an event's age, but instead is dependent on where the boundary is. For example, the amount of telescoping for an event that is 8 weeks old will vary, depending on whether the boundary is in the 11 th week or the 15 th week.

To test this prediction, we took advantage of the fact that one of the authors teaches on the semester system ( 15 weeks of classes plus test week) and another teaches on the quarter system (10 weeks of classes plus test week). In our initial analysis, we calculated for each subject the mean weekly dating error for events dated in each of the first 8 weeks of the academic term. These means were then analyzed in a retention interval $\times$ boundary condition mixed analysis of variance (ANOVA).

The data are shown in Figure 3, and are consistent with the predictions derived from the boundary hypothesis. As can be seen from that figure, the mean dating errors for the two groups are approximately equivalent for the retention intervals up to and including Week 6, but they diverge for Weeks 7 and 8 . The dating errors for the group (OSUN) with the maximum retention interval of 10 weeks (70 days) show substantial apparent telescoping, whereas the dating errors for the group (KSU) with the maximum retention interval of 14 weeks (98 days) do not show telescoping. Given that the data collected on the two campuses were essentially the same except for the boundary, these data suggest that the boundary is producing the telescoping in date estimates.

The data in Figure 3 also suggest that there are slight time expansion effects for events in recent retention intervals, but that these time expansion effects were essentially equivalent for the two boundary groups.

The results of the ANOVA support these conclusions. The overall performance of the two groups differed reliably $\left[F(1,61)=5.53, M S_{\mathrm{e}}=68.74, p<.03\right]$. In addition, there was a reliable effect of retention interval on dating error $\left[F(7,427)=10.67, M S_{\mathrm{e}}=28.54, p<\right.$ $.001]$. Most importantly, the boundary condition $\times$ retention interval interaction was significant $[F(7,427)=4.67$, $\left.M S_{\mathrm{e}}=28.54, p<.001\right]$. Subsequent Tukey tests showed that the groups differed reliably only for retention intervals of 7 and 8 weeks.

A more fine-grained analysis of the data graphically illustrates the regularity of the time expansion and telescoping effects in both groups. The average dating errors by boundary condition are presented in Figure 4 for the entire time frame of the study (each point is the average of 2 consecutive days). As those data indicate, the basic form of the curve is the same for subjects in both groups. There is evidence of slight time expansion (age overestimation) for recent events, and of substantial telescoping (age underestimation) for older events.

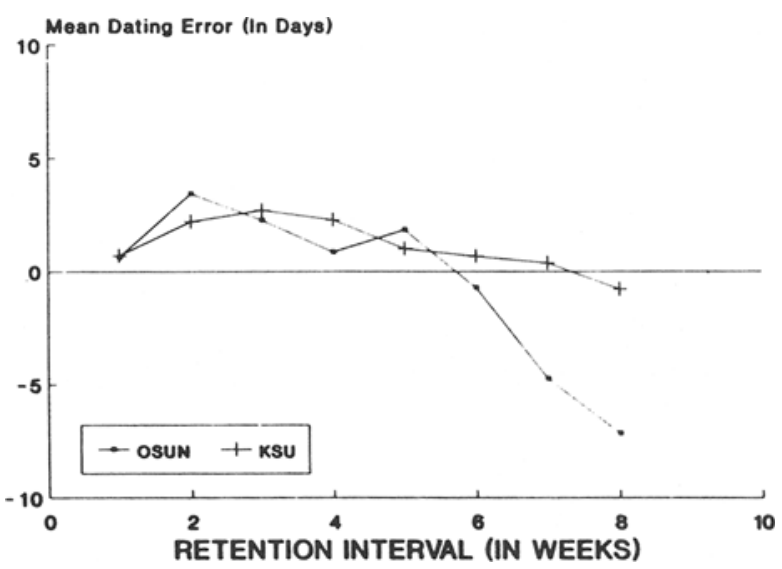

Figure 3. Mean datung error (in days) by retention interval (weekly) for OSUN and KSU subjects. 


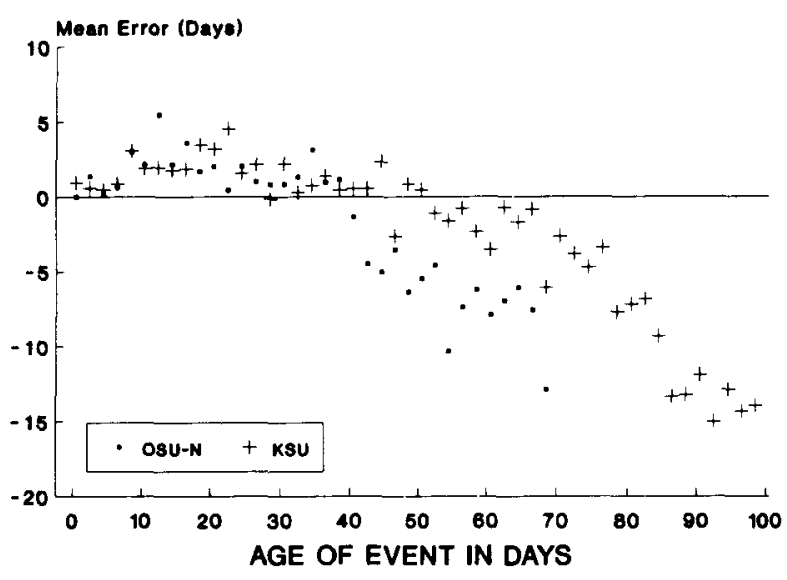

Figure 4. Mean dating error (in days) across the entire retention interval for OSUN and KSU subjects (each point is the average of errors for 2 days).

However, despite this basic overall similarity, it is obvious that age underestimation occurs for events much earlier for subjects in the 10-week boundary condition than in the 15-week boundary condition.

These data lend strong support for the boundary effects suggested by Huttenlocher et al. (1988) and by Rubin and Baddeley (1989). The data collected from the two groups were identical-the only major difference between the two groups was the maximum retention interval. As predicted by boundary theorists, manipulation of boundaries produced differences in telescoping. Furthermore, as predicted by the boundary theorists, both groups of subjects showed modest time expansion effects during Weeks 2-5.

These data are inconsistent with the telescoping mechanism proposed by Thompson et al. (1988b; see also Huttenlocher et al., 1988), who suggested that telescoping might be produced by event memory. More specifically, Thompson et al. suggested that, as a strategy to date an event, people might estimate the number of intervening events that have occurred since the event-the more intervening events recalled, the longer the interval. However, because the passage of time causes people to forget events, people's recollections of events could be relatively sparse, causing a foreshortening of perceptions of time and telescoping of event date estimates. This explanation implies that telescoping should be independent of boundaries, an outcome that was obviously not obtained in the present studies. The boundary data, along with the data from Table 1 indicating that estimating the number of intervening events is not a frequently used strategy, suggests that intervening event estimation plays only a small role in telescoping, if any (Burt, 1992, reaches a similar conclusion for perceptions of event duration).

These data also speak to other dating issues. As indicated earlier, Huttenlocher et al. (1990) asked subjects to estimate event ages, as opposed to our procedure of asking subjects to provide event dates. Despite the dif- ferent methodologies, the pattern of dating errors across time looks remarkably similar in both paradigms. This similarity suggests that bounding effects are fairly general and are independent of the manner in which dating is assessed.

Our data also have implications for the rounding mechanism for telescoping suggested by Huttenlocher et al. (1990). As indicated earlier, we have no evidence of rounding effects when we ask subjects to provide us with event dates. Although rounding obviously occurs (as we found in the error estimates depicted in Figure 2), the fundamental similarity in results obtained with the different dating methodologies suggests that rounding probably has only a minor role to play in the production of telescoping effects.

\section{CONCLUSIONS}

Even in a study offering many advantages that would seem to facilitate recall of event dates (writing events in a diary, a relatively short time frame), the data in the present paper suggest that people can only infrequently recall the exact date of an event, and instead must resort to the use of partial temporal information to reconstruct the event's date. This partial temporal information seems to come from many different sources in memory.

The partial temporal information that subjects use can sometimes be directly recalled. That is, temporal information sometimes seems to take the form of a tag (a date, a day of the week, a month, a year) attached to an event. More often, however, even the partial temporal information contributing to a date has to be inferred from some other aspect of memory: recalled landmarks or boundaries, recalled event sequences, or the nature of the event.

Although a researcher interested in investigating the impact of memory on event dating might be daunted by our evidence suggesting that multiple memory types contribute to event dating, the data presented in this paper should also lead those researchers to be a bit more optimistic about isolating the impact of these various memory types. The data in this paper suggest that the use of certain memories (and associated strategies) leave characteristic "footprints" in the data, and that these footprints are not all that difficult to find. Given our own modest success, we suggest that a productive avenue for future research would be to further identify the types of memory contributing to event dating and to more specifically isolate the impact of those memory types on dating accuracy and error patterns.

\section{REFERENCES}

Bradburn, N. M., Rips, L. J., \& Shevell, S. K. (1987). Answering autobiographical questions: The impact of memory and inference on surveys. Science, 236, 151-167.

BrEwER, W. F. (1988). Memory for randomly sampled autobiographical events. In U. Neisser \& E. Winograd (Eds.), Remembering reconsidered: Ecological and traditional approaches to the study of memory (pp. 21-90). Cambridge: Cambridge University Press. 
Brown, N. R., RiPs, L. J., \& Shevell, S. K. (1985). The subjective dates of natural events in very-long-term memory. Cognitive Psychol ogy, 17, 139-177.

BuRT, C. D. B. (1992). Reconstruction of the duration of autobiographical events. Memory \& Cognition, 20, 124-132.

Huttenlocher, J., Hedges, L. V., \& Bradburn, N. M. (1990) Reports of elapsed time: Bounding and rounding processes in estimation. Journal of Experimental Psychology: Learning, Memory, \& Cognition, 16, 196-213

Hutteniocher, J., Hedges, L., \& Prohaska, V. (1988). Hierarchical organization in ordered domains: Estimating the dates of events. Psychological Review, 95, 471-484.

LofTus, E. F., \& MArburger, W. (1983). Since the eruption of Mt. St. Helens, has anyone beaten you up? Improving the accuracy of retrospective reports with landmark events. Memory \& Cognition, 11, 114-120.

Roenker, D. L., Thompson, C. P., \& Brown, S. C. (1971). A comparison of measures for the estimation of clustering in free recall Psychological Bulletin, 10, 45-48.

RuBIN, D. C., \& BADDELEY, A. D. (1989). Telescoping is not time compression: A model of the dating of autobiographical events. Memory and Cognition, 17, 653-661

Skowronski, J. J., Betz, A. L., Thompson, C. P., \& Shannon, L. (1991). Social memory in everyday life: The recall of self-events and other-events. Journal of Personality \& Social Psychology, 60, 831-843.

Skowronski, J. J., Betz, A. L., Thompson, C. P., Walker, W. R., \& SHANNON, L. (in press). The impact of differing memory domains on event dating processes in self and proxy reports. In S. Sudman \& N. Schwartz (Eds.), Autobiographical memory and the validity of retrospective reports. New York: Springer-Verlag.

Thompson, C. P. (1982). Memory for unique personal events: The roommate study. Memory \& Cognition, 10, 324-332.

Thompson, C. P. (1985). Memory for unique personal events: Some implications of the self-schema. Human Learning, 4, 267-280.

Thompson, C. P., \& RoEnKer, D. L. (1971). Learning to cluster. Journal of Experimental Psychology, 91, 136-139.
Thompson, C. P., Skowronski, J. J., \& Lee, D. J. (1988a). Reconstructing the date of a personal event. In M. M. Gruneberg. P. E. Morris, \& R. N. Sykes (Eds.), Practical aspects of memory: Current research and issues (Vol. 1, pp. 241-246). New York: Wiley.

Thompson, C. P., Skowronski, J. J., \& Lee, D. J. (1988b). Telescoping in dating naturally occurring events. Memory \& Cognition, 16, 461-468.

WAGENAAR, W. A. (1986). My memory: A study of autobiographical memory over six years. Cognitive Psychology, 18, 225-252.

\section{NOTES}

1. This theoretical distribution has an empirical basis. Using the standard deviations and mean error values at each actual event age value, one can calculate a probability that a subject will make a dating error of a particular magnitude for events of that age. By summing these probabilities across all event ages, one can come up with an overall estimate of the probability of making an error of a given magnitude. We have conducted several such runs on old data sets, and the general shape of the obtained curve is similar to the theoretical exponential curve that we use as the basis for our model.

2. As indicated in the text, our assumption of a $10 \%$ exact date rate was derived from the results of previous studies done with this type of paradigm. However, this does not necessarily imply that this $10 \%$ figure will always be appropriate. For example, for longer dating periods, one might expect the frequency with which subjects will use this strategy to go down; for shorter dating periods, that frequency might go up. We settled on the $10 \%$ figure for this study because it was most justifiable in light of our past research, and we did not feel that it was appropriate to use the percentage in the present data $(17 \%)$ as the estimate for constructing our model-it would tend to exaggerate the apparent fit between the model and the data.

3 . This possibility was raised by one of the reviewers of this paper.

(Manuscript received April 6, 1992; revision accepted for publication September 17,1992 .) 